\title{
Microbial aetiology of community-acquired pneumonia and its relation to severity
}

\author{
Catia Cillóniz, ${ }^{1,5}$ Santiago Ewig, ${ }^{2}$ Eva Polverino, ${ }^{1,5}$ Maria Angeles Marcos, ${ }^{3}$ \\ Cristina Esquinas, ${ }^{1}$ Albert Gabarrús, ${ }^{1}$ Josep Mensa, ${ }^{4}$ Antoni Torres ${ }^{1,5}$
}

\begin{abstract}
- Additional data are published online only. To view these files please visit the journal online (http://thorax.bmj.com).

${ }^{1}$ Servei de Pneumologia, Institut del Tòrax, Hospital Clinic, IDIBAPS, Universitat de Barcelona, Barcelona, Spain 2Department of Pneumology Thoraxzentrum Ruhrgebiet, Herne und Bochum, Germany ${ }^{3}$ Department of Microbiology, Hospital Clínic, Barcelona, Spain ${ }^{4}$ Department of Infectious Diseases, Hospital Clínic, Barcelona, IDIBAPS, Spain ${ }^{5}$ Centro de Investigación Biomédica En Red-Enfermedades Respiratorias (CibeRes, CB06/06/0028), Instituto de Salud Carlos III, Ministerio de Ciencia e Innovación, Spain
\end{abstract}

\section{Correspondence to}

Antoni Torres, Servei de Pneumologia, Institut Clínic de Pneumologia i Cirurgia Toràcica, Hospital Clinic, Villarroel 170, Barcelona 08036, Spain; atorres@ub.edu

Received 28 May 2010 Accepted 9 December 2010 Published Online First 21 January 2011

\section{ABSTRACT}

Background The distribution of the microbial aetiology and mortality of community-acquired pneumonia (CAP) was investigated in relation to the clinical setting and severity scores (pneumonia severity index (PSI) and confusion, blood urea nitrogen, respiratory rate, blood pressure, age (CURB-65)).

Methods 3523 patients with CAP were included $115 \%$ outpatients, $85 \%$ inpatients). The distribution of the microbial aetiology in relation to the clinical setting and severity scores (PSI, CURB-65) and the relative mortality of different aetiologies across the severity scores were analysed.

Results The aetiology was established in 1463 patients (42\%), of whom 257 died (7\%). The ranking of aetiologies varied according to site of care, with increasing frequency of Streptococcus pneumoniae and mixed aetiologies and decreasing frequency of atypical pathogens in hospitalised patients and those in ICUs. The distribution of aetiologies according to severity scores showed corresponding patterns; however, the severity scores were more sensitive to Gram-negative enteric bacilli (GNEB) and Pseudomonas aeruginosa and less sensitive in identifying mixed aetiologies as moderateand high-risk conditions. Mortality rates according to aetiology and severity scoring showed increasing mortality rates for all pathogens except atypical pathogens. $S$ pneumoniae had the highest number of deaths while GNEB, $P$ aeruginosa, Staphylococcus aureus and mixed aetiologies had the highest mortality rates. Legionella pneumophila was similarly distributed according to site of care and prognostic scores.

Conclusions CAP due to atypical bacterial pathogens is recognised both clinically and by severity scoring as a low-risk condition. Severity scores are more sensitive in identifying patients with GNEB and $P$ aeruginosa as moderate- and high-risk aetiologies whereas mixed aetiologies may be underestimated.

\section{INTRODUCTION}

Despite the use of many microbiological techniques, in nearly $50 \%$ of cases of communityacquired pneumonia (CAP) the aetiology is unknown. ${ }^{1}$ Most studies show that Streptococcus pneumoniae is the most common pathogen in CAP, and in hospitalised patients it accounts for twothirds of the mortality. ${ }^{2}$ Other bacterial agents including Haemophilus influenzae and atypical pathogens (Mycoplasma pneumoniae, Chlamydophila pneumoniae, Coxiella burnetii and Legionella pneumophila) are described in up to $35 \%$ of CAP episodes. In addition, viral pathogens are recognised as causes of CAP, with influenza virus the leading viral pathogen. $^{13}$

Since an aetiological diagnosis of CAP is usually unavailable, the initial empirical treatment is usually guided by microbial patterns described for the risk categories. ${ }^{4}{ }^{5}$ In general, the guidelines for CAP management advocate empirical antibiotic selection on the basis of the age of the patient, comorbidities and initial severity of the illness. ${ }^{6-8}$

Recommendations of initial antimicrobial treatment according to pneumonia severity are mainly based on the notion that the risk of adverse outcomes including excess mortality in patients receiving inadequate initial antimicrobial treatment increases with pneumonia severity. However, few studies have assessed the independent effect of the microbial aetiology on severity scores (pneumonia severity index (PSI) and confusion, blood urea nitrogen, respiratory rate, blood pressure, age (CURB-65)). ${ }^{9} 10$ This study therefore aimed to investigate the distribution of the aetiology according to clinical setting and severity scores (PSI and CURB-65) and the relative mortality rates in a large series of patients with CAP.

\section{METHODS}

\section{Study setting and design}

All consecutive adult patients attending the Hospital Clinic in Barcelona with CAP were prospectively studied between November 1996 and July 2008. CAP was defined as the presence of a new infiltrate on chest radiography together with clinical symptoms suggestive of lower respiratory tract infection. Exclusion criteria included: (1) severe immunosuppression (AIDS, chemotherapy, immunosuppressive drugs); (2) active tuberculosis (3) healthcare-associated pneumonia; and (4) cases with a confirmed alternative diagnosis. Nonhospitalised patients were visited $1-7$ days after attending the outpatient clinic.

\section{Patient characteristics and CAP scoring systems}

The following data were recorded on admission to hospital: age, gender, current smoking, comorbid illnesses and antimicrobial treatment prior to hospital admission, duration of symptoms prior to visit, clinical symptoms, physical examination, chest $\mathrm{x}$-ray pattern, blood analysis and antimicrobial treatment at admission. All surviving patients were visited 30-40 days after discharge. PSI and CURB-65 score classes were assigned according to the authors' original designations. Patients were stratified into low-risk, intermediate-risk and highrisk classes as follows: PSI score: low risk=classes 
I-III, intermediate risk=class IV, high risk=class V; CURB-65: low risk $=$ classes $0-1$, intermediate risk $=$ class 2 , high risk $=$ classes 3-5.

\section{Microbiological evaluation}

Microbiological examination was performed in sputum, urine, two samples of blood and nasopharyngeal swabs. Pleural puncture, tracheobronchial aspirates (TBAS) and bronchoalveolar lavage (BAL) fluid, when available, were collected for Gram and Ziehl-Nielsen stains and for cultures for bacterial, fungal and mycobacterial pathogens.

The aetiology was considered definite if one of the following criteria was met: (1) positive blood culture (in the absence of an apparent extrapulmonary focus); (2) positive bacterial culture of pleural fluid or transthoracic needle aspiration samples; (3) elevated serum levels of IgM against $C$ pneumoniae ( $\geq 1: 64), C$ burnetii ( $\geq 1: 80$ ) and $M$ pneumoniae (any positive titre); (4) positive urinary antigen for $L$ pneumophila (Binax Now L pneumophila urinary antigen test; Trinity Biotech, Bray, Ireland); (5) positive urinary antigen for $S$ pneumoniae (Binax Now $S$ pneumoniae urinary antigen test; Emergo Europe, The Netherlands); (6) bacterial growth in cultures of TBAS $\left(\geq 10^{5} \mathrm{cfu} / \mathrm{ml}\right)$, protected specimen brushing (PSB) $\left(\geq 10^{3} \mathrm{cfu} / \mathrm{ml}\right)$ and BAL fluid $\left(\geq 10^{4} \mathrm{cfu} / \mathrm{ml}\right)$; 7 ) seroconversion (ie, a fourfold increase in IgG titres) for $C$ pneumoniae and $L$ pneumophila $>1: 128, C$ burnetii $>1: 80$ and respiratory viruses (influenza viruses $A$ and $B$, parainfluenza viruses $1-3$, respiratory syncytial virus, adenovirus); (8) detection of antigens by immunofluorescence assay plus virus isolation or detection by reverse transcriptase (RT)-PCR testing for respiratory viruses (influenza viruses $A$ and $B$, parainfluenza viruses $1-3$, respiratory syncytial virus, adenovirus).

The aetiology of pneumonia was classified as presumptive when a predominant microorganism was isolated from a purulent sample (leucocytes $>25$ per high power microscopic field and few epithelial cells $<10$ per high power microscopic field) and the findings of Gram staining were compatible. For the purpose of this study, presumptive and definitive diagnoses were analysed together.

\section{Statistical analysis}

Categorical variables were described with counts and percentages. Data for continuous variables were presented as mean (SD) or median (IOR) where appropriate. The proportions of individuals in each of the sites of care and PSI and CURB-65 groups diagnosed with each pathogen of interest were compared using $\chi^{2}$ tests. $\chi^{2}$ tests were also used to compare the proportions of individuals with each pathogen of interest between each pair of site of care, PSI and CURB-65 groups, with $p$ values adjusted using the Bonferroni method. The $\kappa$ concordance coefficient was calculated to determine agreement with risk class assignment. The interpretation of the $\mathrm{K}$ index value was based on the Altman scale $(<0.20$, poor; 0.21-0.40, fair; 0.41-0.60, moderate; $0.61-0.80$, good; and $0.81-1.00$, very good). ${ }^{11}$ All analyses were performed with SPSS software Version 16; a two-sided $p$ value of $<0.05$ was considered statistically significant.

\section{RESULTS}

Patient characteristics and outcomes

A total of 3523 adult patients with CAP were studied, of whom $514(14.6 \%)$ were outpatients. The mean (SD) age was 65.5 (18.7) years (range 18-102); 2166 patients (62\%) were aged $\geq 65$ years. The main clinical characteristics and radiological findings of the study population are shown in table 1 .
Table 1 Main characteristics of study population

\begin{tabular}{ll}
\hline Characteristic & $\mathbf{n ~ ( \% )}$ \\
\hline Total number of patients & 3523 \\
Demographic data & \\
Mean (SD) age (years) & $65.5(18.7)$ \\
Male & $2218(63)$ \\
History of smoking & $963(28)$ \\
History of alcohol abuse & $581(17)$ \\
Site of care & \\
Outpatients & $514(15)$ \\
Inpatients & $3009(85)$ \\
$\quad$ Ward & $2521(72)$ \\
$\quad$ ICU & $488(14)$ \\
Comorbidities & \\
Chronic respiratory disease & $1599(46)$ \\
Chronic cardiovascular disease & $640(18)$ \\
Diabetes mellitus & $538(16)$ \\
Neurological disease & $555(16)$ \\
Chronic renal disease & $247(7)$ \\
Chronic hepatic disease & $154(4)$ \\
Symptoms & \\
Cough & $2827(81)$ \\
Sputum & $2098(61)$ \\
Dyspnoea & $2327(67)$ \\
Chest pain & $1435(41)$ \\
Fever & $2824(81)$ \\
Median (IQR) duration of symptoms (days) & $4.0(5.0)$ \\
Clinical, laboratory and radiographic findings & \\
Median (IQR) CRP (mg/dl) & $17.0(19.3)$ \\
Median (IQR) WBC count ( $\times 10^{9} /$ ) & $13.0(8.3)$ \\
Median (IOR) platelet count ( $\times 10^{9} /$ ) & $237.0(115.0)$ \\
Alveolar pattern infiltrates & $2920(87)$ \\
Multilobar involvement ( $\geq 2$ lobes) & $844(24)$ \\
Pleural effusion & $485(14)$ \\
Median (IQR) length of hospital stay (days) & $6.0(6.0)$ \\
\hline CRP, C-reactive protein; ICU, intensive care unit; WBC, white blood cells. \\
& \\
&
\end{tabular}

A total of 769 patients $(22.4 \%)$ had received antimicrobial treatment prior to hospital admission. One-month mortality was $7 \%(n=257)$.

The distribution of groups by severity scores (PSI, CURB-65) and by site of care is shown in table 2 . On admission, both scores placed the majority of patients in the low-risk group (PSI $46 \%$; CURB-65 51\%). Similarly, both scores classified similar proportions in the high-risk group (PSI, 20\%; CURB-65, 21\%). Nevertheless, the accordance of risk class assignment was moderate $(\kappa=0.54, \mathrm{p}<0.001)$ : PSI classes I-III and CURB-65 classes $0-1$ ( $n=1404$ patients), PSI class IV and CURB-65 class $2(n=606)$ and PSI class V and CURB-65 classes $3-5(n=479)$. Predictions of death were similar for both risk scores (risk group 1, 1.2\% and $1.8 \%$; risk group 2, $6.8 \%$ and $7.9 \%$; risk group $3,21.5 \%$ and $19.2 \%)$. However, the accordance of predictions was low $(\kappa=0.37, \mathrm{p}<0.001)$ : PSI classes I-III and CURB-65 classes $0-1$ $(n=10)$, PSI class IV and CURB-65 class $2(n=41)$ and PSI class V and CURB-65 classes 3-5 $(n=114)$.

\section{Diagnostic yield of applied techniques}

Aetiological diagnoses were established in 1463 cases (42\%). Overall, 1463 pathogens were identified, of which 490 (34\%) were detected by sputum examination (presumptively diagnostic) and $973(66 \%)$ by other techniques (definitively diagnostic). A single pathogen was identified in 1255 patients (86\%) and two pathogens were identified in 208 (14\%). Additional details of all applied techniques (number of performed tests and diagnostic rates) are provided in the online supplement. 
Table 2 Distribution of patients and 30-day mortality in each risk class assessed using the PSI and CURB-65 scores and by site of care

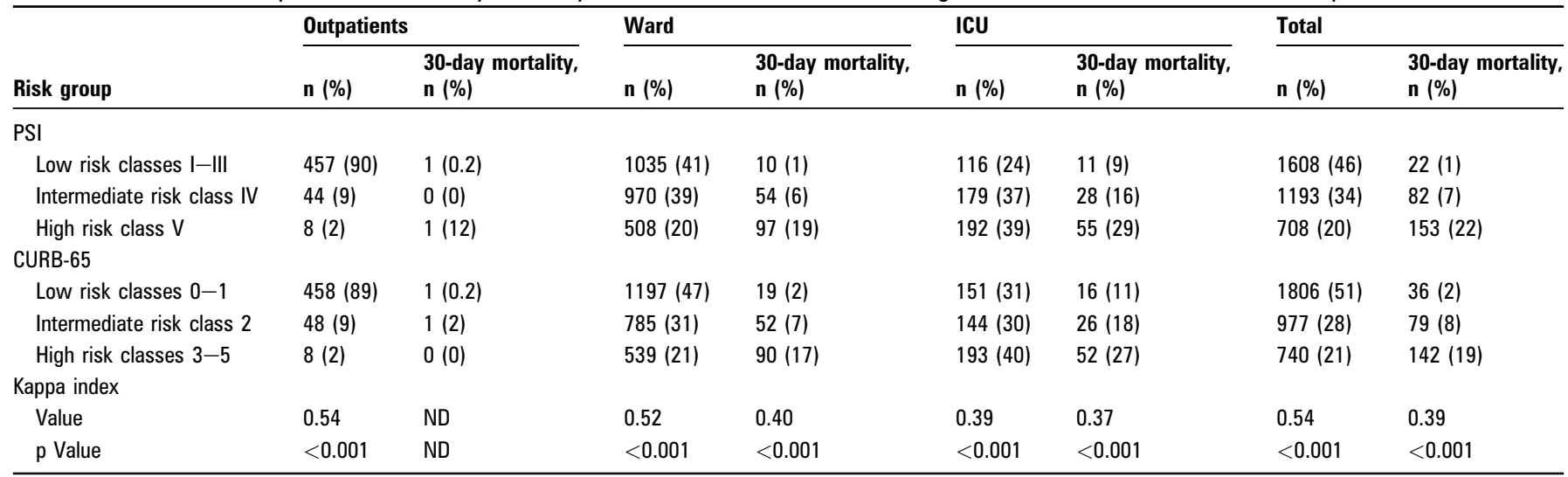

30-day mortality calculated as the ratio between the total number of patient deaths in each risk class and the total number of patients in each risk class multiplied by 100 . PSI was not available in 14 patients.

CURB-65, confusion, blood urea nitrogen, respiratory rate, blood pressure, age; ND, not done; PSI, pneumonia severity index.

\section{Microbial aetiology of patients with CAP}

The five most frequently isolated pathogens were $S$ pneumoniae (613 patients, 42\%), atypical pathogens ( $C$ pneumoniae, $M$ pneumoniae, L pneumophila and C burnetii) (263 patients, 18\%), viruses (148 patients, 10\%), $H$ influenzae (70 patients, $5 \%$ ) and Pseudomonas aeruginosa (50 patients, 3\%).

More than one causative agent was found in 208 patients (14\%); S pneumoniae was the most prevalent microorganism involved in mixed infections $(136 / 208,65 \%)$. The most frequent combinations among mixed infections were: two bacteria in 67 $(32 \%)$, a bacterium plus a virus in $61(29 \%)$ and a bacterium plus an atypical pathogen in 37 (18\%) (see online supplement).

Figure 1 in the online supplement illustrates the rate of pathogen isolation during the 12 -year period, showing a homogeneous incidence of all pathogens over time except for an increase in $S$ pneumoniae in 2000 and in respiratory viruses in 2004 as a result of the introduction of the pneumococcal urinary antigen test and RT-PCR for viruses, respectively.

Overall, 333 patients had bacteraemia (S pneumoniae in 265 patients, GNEB in 14, Staphylococcus aureus in 13, H influenzae in 7, $P$ aeruginosa in 2 and other streptococci in 32 patients). The mortality rate associated with bacteraemic pneumococcal pneumonia was $11 \%$.

\section{Microbial aetiology by site of care}

The microbial aetiology was known in $32 \%$ of outpatients (514 isolates) and in 44\% of inpatients (1302 isolates). The yield was 1042 of 2521 (41\%) in patients treated on the ward and 260 of $488(53 \%)$ in those treated in the ICU (table 3$)$. The most frequent aetiology among outpatients was the atypical pathogen group (36\%) followed by $S$ pneumoniae (35\%), viruses and mixed aetiologies (both 9\%). In patients treated on the ward, $S$ pneumoniae was the most common aetiology (43\%) followed by atypical pathogens (16\%), mixed aetiologies (13\%) and viruses (12\%). L pneumophila was identified in 10 patients $(6 \%)$. In patients treated in the ICU the most common aetiologies were $S$ pneumoniae (42\%), mixed aetiologies (22\%) and atypical pathogens (14\%).

\section{Microbial aetiology according to severity scores}

When we analysed the aetiology by severity score groups (tables 4 and 5), the most common pathogen was $S$ pneumoniae in all risk groups. In patients with low-risk scores, atypical pathogens were second (25\%) and mixed aetiologies third (13\%). The frequency of atypical pathogens decreased with severity
(15\% and $8 \%$, respectively), whereas that of mixed aetiologies increased (15\% and $17 \%$, respectively). The frequencies of $P$ aeruginosa and GNEB increased in higher risk classes $(3 \%$ and $8 \%$, and $2 \%$ and $4 \%$ ). Corresponding trends were found in the distributions according to the CURB-65 severity score.

\section{Mortality according to severity scores and microbial aetiology} Mortality rates of specific aetiologies per PSI score revealed increasing mortality rates with increasing severity scores for most pathogens. S pneumoniae had the highest number of deaths. High mortality rates were observed for $S$ aureus and GNEB (11\% and $14 \%$, respectively) in low-risk classes. The highest mortality rates in the group treated on the ward had GNEB $(26 \%)$, Moraxella catarrhalis (25\%), S aureus (11\%) and $P$ aeruginosa $(11 \%)$. In patients treated in the ICU, $S$ aureus (67\%), GNEB (67\%), P aeruginosa (33\%) and mixed aetiologies (24\%) had the highest mortalities. Again, similar patterns were found for the CURB-65 score (tables 6 and 7).

\section{DISCUSSION}

The main findings of this study are: (1) according to the site of care, the ranking of aetiologies varies with an increase in mixed aetiologies and decreasing frequency of atypical pathogens from ward to ICU; (2) the distribution of aetiologies according to both severity scores PSI and CURB-65 revealed corresponding patterns; (3) mortality rates by aetiology and severity scoring showed increasing mortality rates for all pathogens except atypical pathogens. $S$ pneumoniae had the highest number of deaths, whereas GNEB, $P$ aeruginosa, $S$ aureus, mixed aetiologies and others had the highest mortality rates.

Several recent studies have shown that PSI and CURB-65 (and its modification CRB-65) yield similar predictions of in-hospital mortality in patients with CAP. ${ }^{12-14}$ Our data support this finding, clearly showing a pattern of three risk classes of low-, moderate- and high-risk with mortality rates of approximately $1-2 \%, 7-8 \%$ and $19-22 \%$, respectively. The last figure has been reported to be higher (30-35\%) when nursing home and bedridden patients are included. ${ }^{15} 16$ However, the severity scores do not measure the same factors since accordance rates are only moderate. Furthermore, it is unclear what these severity scores truly reflect, so it seems highly relevant to study the relation between aetiology and severity.

This study is novel in that it includes three dimensions of analysis: the relation of aetiology to site of care as a reflection of clinical judgement of severity and other considerations; its 
Table 3 Aetiology of community-acquired pneumonia by site of care (number of patients with known aetiology in specific site of care)

\begin{tabular}{|c|c|c|c|c|c|}
\hline Aetiology & $\begin{array}{l}\text { Outpatients } \\
(n=161)\end{array}$ & $\begin{array}{l}\text { Ward } \\
(n=1042)\end{array}$ & $\begin{array}{l}\text { ICU } \\
(n=260)\end{array}$ & $\begin{array}{l}\text { Total } \\
(n=1463)\end{array}$ & p Value \\
\hline Streptococcus pneumoniae & $56(35)$ & $447(43)$ & $110(42)$ & $613(42)$ & 0.150 \\
\hline Haemophilus influenzae & $8(5)$ & $54(5)$ & $8(3)$ & $70(5)$ & 0.361 \\
\hline Moraxella catarrhalis & $0(0)$ & $4(0.4)$ & $1(0.4)$ & $5(0.3)$ & 0.733 \\
\hline Atypical bacteria* $†$ & $58(36)$ & $168(16)$ & $37(14)$ & $263(18)$ & $<0.001$ \\
\hline Legionella pneumophila & $10(6)$ & $87(8)$ & $21(8)$ & $118(8)$ & 0.650 \\
\hline Mycoplasma pneumoniae ${ }^{*} \dagger$ & $27(17)$ & $32(3)$ & $6(2)$ & $65(4)$ & $<0.001$ \\
\hline Chlamydophila pneumoniae & $10(6)$ & $32(3)$ & $8(3)$ & $50(3)$ & 0.117 \\
\hline Coxiella burnetii* $†$ & $11(7)$ & $17(2)$ & $2(1)$ & $30(2)$ & $<0.001$ \\
\hline Virus $\ddagger$ & $15(9)$ & $123(12)$ & $10(4)$ & $148(10)$ & $<0.001$ \\
\hline Staphylococcus aureus & $1(1)$ & $18(2)$ & $6(2)$ & $25(2)$ & 0.429 \\
\hline MS & $1(1)$ & $9(1)$ & $4(2)$ & $14(1)$ & 0.545 \\
\hline MR & $0(0)$ & $9(1)$ & $2(1)$ & $11(1)$ & 0.498 \\
\hline GNEB & $1(1)$ & $23(2)$ & $3(1)$ & $27(2)$ & 0.250 \\
\hline Pseudomonas aeruginosa & $1(1)$ & $37(4)$ & $12(5)$ & $50(3)$ & 0.082 \\
\hline Mixed $\dagger \neq$ & $15(9)$ & $135(13)$ & $58(22)$ & $208(14)$ & $<0.001$ \\
\hline Others & $6(4)$ & $33(3)$ & $15(6)$ & $54(4)$ & 0.138 \\
\hline $\begin{array}{l}\text { Data are expressed as } n(\%) \text {. } \\
{ }^{*} p<0.005 \text {, outpatients vs ward. } \\
\dagger p<0.005 \text {, outpatients vs ICU. } \\
\neq p<0.005 \text {, ward vs ICU. } \\
\text { GNEB, Gram-negative enteric bac }\end{array}$ & & & & & \\
\hline
\end{tabular}

relation to risk classes according to both scores; and its relation to mortality according to risk classes.

When analysing the relation of aetiology to site of care, patients with the two atypical bacterial pathogens $M$ pneumoniae and $C$ burnetii were significantly more frequently treated as outpatients. This is an expected finding since CAP due to $M$ pneumoniae and $C$ pneumoniae are usually encountered in younger patients without comorbidity and has a mild clinical course. $^{3} 1718$ The severity scores equally showed that $M$ pneumoniae and $C$ burnetii were found more frequently in low-risk patients (and a similar trend for $C$ pneumoniae). Accordingly, mortality was minimal in this group, regardless of the risk class.
L pneumophila was equally distributed across all sites of care and all risk classes, with the exception of a significantly higher frequency of patients in the moderate-risk group according to the PSI. Since the association with severity was not obvious in the high-risk group, this finding cannot be considered as a consistent trend. These findings do not support those of previous studies from the same region ${ }^{9}{ }^{10}$ which show low rates of Legionella spp. in patients with less severe disease. The equal distribution may be partly explained by an unexpected low overall mortality in patients with this pathogen. Indeed, the mortality of Legionella pneumonia has decreased over the years since the implementation of urinary antigen detection. ${ }^{19}$

Table 4 Aetiology according to severity score (PSI) (number of patients with known aetiology in specific risk class)

\begin{tabular}{|c|c|c|c|c|c|}
\hline Aetiology & $\begin{array}{l}\text { PSI I-III } \\
(n=659)\end{array}$ & $\begin{array}{l}\text { PSI IV } \\
(n=500)\end{array}$ & $\begin{array}{l}\text { PSI V } \\
(n=301)\end{array}$ & $\begin{array}{l}\text { Total } \\
(n=1460)\end{array}$ & p Value \\
\hline Streptococcus pneumoniae & $276(42)$ & $205(41)$ & $132(44)$ & $613(42)$ & 0.728 \\
\hline Haemophilus influenzae & $27(4)$ & $28(6)$ & $15(5)$ & $70(5)$ & 0.488 \\
\hline Moraxella catarrhalis & $2(0.3)$ & $2(0.4)$ & $1(0.3)$ & $5(0.3)$ & 0.961 \\
\hline Atypical bacteria* $\dagger \neq$ & $163(25)$ & $77(15)$ & $23(8)$ & $263(18)$ & $<0.001$ \\
\hline Legionella pneumophila & $54(8)$ & $50(10)$ & $14(5)$ & $118(8)$ & 0.027 \\
\hline Mycoplasma pneumoniae ${ }^{*} \dagger$ & $51(8)$ & $12(2)$ & $2(1)$ & $65(4)$ & $<0.001$ \\
\hline Chlamydophila pneumoniae & $31(5)$ & $13(3)$ & $6(2)$ & $50(3)$ & 0.046 \\
\hline Coxiella burnetii* $†$ & $27(4)$ & $2(0.4)$ & $1(0.3)$ & $30(2)$ & $<0.001$ \\
\hline Virus & $62(9)$ & $57(11)$ & $29(10)$ & $148(10)$ & 0.511 \\
\hline Staphylococcus aureus & $9(1)$ & $10(2)$ & $6(2)$ & $25(2)$ & 0.651 \\
\hline MS & $5(1)$ & $5(1)$ & $4(1)$ & $14(1)$ & 0.697 \\
\hline MR & $4(1)$ & $5(1)$ & $2(1)$ & $11(1)$ & 0.731 \\
\hline GNEB $†$ & $7(1)$ & $9(2)$ & $11(4)$ & $27(2)$ & 0.022 \\
\hline Pseudomonas aeruginosa $\dagger \neq$ & $9(1)$ & $17(3)$ & $23(8)$ & $49(3)$ & $<0.001$ \\
\hline Mixed & $84(13)$ & $73(15)$ & $51(17)$ & $208(14)$ & 0.217 \\
\hline Others & $20(3)$ & $22(4)$ & $10(3)$ & $52(4)$ & 0.448 \\
\hline $\begin{array}{l}\text { Data are expressed as } n(\%) . \\
\text { PSI was not available in three pa } \\
{ }^{*} p<0.005 \text {, PSI classes I-III vs } \\
\dagger p<0.005 \text {, PSI classes I-III vs } \\
\neq p<0.005 \text {, PSI class IV vs class }\end{array}$ & & & & & \\
\hline
\end{tabular}


Table 5 Aetiology according to severity score (CURB-65) (number of patients with known aetiology in specific risk class)

\begin{tabular}{|c|c|c|c|c|c|}
\hline Aetiology & $\begin{array}{l}\text { CURB-65 } 0-1 \\
(n=741)\end{array}$ & $\begin{array}{l}\text { CURB-65 } 2 \\
(n=414)\end{array}$ & $\begin{array}{l}\text { CURB-65 } 3-5 \\
(n=308)\end{array}$ & $\begin{array}{l}\text { Total } \\
(n=1463)\end{array}$ & p Value \\
\hline Streptococcus pneumoniae & $291(39)$ & $180(43)$ & $142(46)$ & $613(42)$ & 0.092 \\
\hline Haemophilus influenzae & $32(4)$ & $23(6)$ & $15(5)$ & $70(5)$ & 0.638 \\
\hline Moraxella catarrhalis & $1(0.1)$ & $1(0.2)$ & $3(1)$ & $5(0.3)$ & 0.097 \\
\hline Atypical bacteria* $† \neq$ & $182(25)$ & $58(14)$ & $23(7)$ & $263(18)$ & $<0.001$ \\
\hline Legionella pneumophila & $65(9)$ & $36(9)$ & $17(6)$ & $118(8)$ & 0.182 \\
\hline Mycoplasma pneumoniae ${ }^{*} \dagger$ & $55(7)$ & $7(2)$ & $3(1)$ & $65(4)$ & $<0.001$ \\
\hline Chlamydophila pneumoniae $†$ & $36(5)$ & $12(3)$ & $2(1)$ & $50(3)$ & 0.002 \\
\hline Coxiella burnetii ${ }^{*} \dagger$ & $26(4)$ & $3(1)$ & $1(0.3)$ & $30(2)$ & $<0.001$ \\
\hline Virus & $81(11)$ & $43(10)$ & $24(8)$ & $148(10)$ & 0.301 \\
\hline Staphylococcus aureus & $8(1)$ & $11(3)$ & $6(2)$ & $25(2)$ & 0.131 \\
\hline $\mathrm{MS}^{*} \dagger$ & $2(0.3)$ & $7(2)$ & $5(2)$ & $14(1)$ & 0.024 \\
\hline MR & $6(1)$ & $4(1)$ & $1(0.3)$ & $11(1)$ & 0.594 \\
\hline GNEB $† \ddagger$ & $10(1)$ & $4(1)$ & $13(4)$ & $27(2)$ & 0.002 \\
\hline Pseudomonas aeruginosa ${ }^{*} \dagger$ & $11(1)$ & $18(4)$ & $21(7)$ & $50(3)$ & $<0.001$ \\
\hline Mixed & $101(14)$ & $61(15)$ & $46(15)$ & $208(14)$ & 0.806 \\
\hline Others & $24(3)$ & $15(4)$ & $15(5)$ & $54(4)$ & 0.441 \\
\hline
\end{tabular}

Data are expressed as $n(\%)$

${ }^{*} \mathrm{p}<0.005$, CURB- 65 classes $0-1$ vs 2 .

$+\mathrm{p}<0.005$, CURB-65 classes $0-1$ vs $3-5$

$\neq \mathrm{p}<0.005$, CURB- 65 class 2 vs $3-5$.

GNEB, Gram-negative enteric bacilli; MR, methicillin-resistant; MS, methicillin-sensitive.

However, in a population with high mortality in the high-risk group, the distribution of severity and, accordingly, of outpatients and inpatients was similar. ${ }^{20}$

Patients infected with viruses were treated less frequently in the ICU and had a very low mortality, although they were placed equally frequently across all risk classes for both severity scores. Since this is a somewhat heterogeneous group it should be regarded with caution, although it agrees with previous findings. ${ }^{21}$

As expected, all pathogens except atypical pathogens showed increasing mortality with increasing disease severity. $S$ pneumoniae had the highest number of deaths, although the relative mortality rates were higher for $S$ aureus, GNEB, Paeruginosa and mixed aetiologies. It therefore seems particularly important to identify these patients.
CAP caused by GNEB and P aeruginosa has been reported to be associated with excess mortality. ${ }^{22}{ }^{23}$ Our data confirm this finding. Both severity scores reflected the adverse prognostic potential of these pathogens since they occur more frequently in moderate- and high-risk groups. Interestingly, no such association could be found in the analysis by site of care and a corresponding trend was only obvious for Paeruginosa. This finding may reflect an underestimation of these pathogens or possibly that some elderly and severely disabled patients were not admitted to the ICU due to prognostic considerations despite a moderate to high risk (PSI, CURB-65) and/or the presence of such aetiologies. ${ }^{23}$

Mixed aetiologies had a high mortality. Importantly, in $32.2 \%$ of our mixed cases, bacteria were associated with other bacteria and bacteria and viruses were found in $29.3 \%$. In fact, de Roux et al found a higher percentage of shock (18\%) when two

Table 6 Mortality from community-acquired pneumonia according to aetiology and PSI risk class: number of deaths among patients with specific aetiology (\% of all deaths with specific aetiology) per PSI risk class

\begin{tabular}{|c|c|c|c|c|}
\hline Aetiology & PSI I-III $(n=22 / 1608,1.4 \%)$ & PSI IV (n=82/1193, 6.9\%) & PSI V $(n=153 / 708,21.6 \%)$ & Total $(n=257 / 3509,7.3 \%)$ \\
\hline Streptococcus pneumoniae & 4/276 (1) & $11 / 205(5)$ & $30 / 132(23)$ & $45 / 613(7)$ \\
\hline Haemophilus influenzae & $0 / 27(0)$ & $0 / 28(0)$ & 2/15 (13) & $2 / 70(3)$ \\
\hline Moraxella catarrhalis & $1 / 2(50)$ & $0 / 2(0)$ & $1 / 1(100)$ & $2 / 5(40)$ \\
\hline Legionella pneumophila & $0 / 54(0)$ & $1 / 50(2)$ & $1 / 14(7)$ & $2 / 118(2)$ \\
\hline Mycoplasma pneumoniae & $1 / 51(2)$ & $1 / 12(8)$ & $0 / 2(0)$ & $2 / 65(3)$ \\
\hline Chlamydophila pneumoniae & $0 / 31(0)$ & $0 / 13(0)$ & $0 / 6(0)$ & $0 / 50(0)$ \\
\hline Staphylococcus aureus & $1 / 9(11)$ & $2 / 10(20)$ & $3 / 6(50)$ & $6 / 25(24)$ \\
\hline MS & $1 / 5(20)$ & $1 / 5(20)$ & $2 / 4(50)$ & $4 / 14(29)$ \\
\hline MR & $0 / 4(0)$ & $1 / 5(20)$ & $1 / 2(50)$ & $2 / 11(18)$ \\
\hline GNEB & $1 / 7(14)$ & $3 / 9(33)$ & $4 / 11(36)$ & $8 / 27(30)$ \\
\hline Pseudomonas aeruginosa & $0 / 9(0)$ & $2 / 17(12)$ & $6 / 23(26)$ & $8 / 49(16)$ \\
\hline
\end{tabular}

Data are expressed as number of deaths/n (\%).

PSI was not available in 14 patients.

GNEB, Gram-negative enteric bacilli; MR, methicillin-resistant; MS, methicillin-sensitive; PSI, pneumonia sensitivity index. 
Table 7 Mortality from community-acquired pneumonia according to aetiology and CURB-65 risk class: number of deaths among patients with specific aetiology (\% of all deaths with specific aetiology) per CURB-65 risk class

\begin{tabular}{|c|c|c|c|c|}
\hline Aetiology & $\begin{array}{l}\text { CURB-65 0-1 } \\
\text { ( } n=36 / 1806,2.0 \%)\end{array}$ & $\begin{array}{l}\text { CURB-65 } 2 \\
(n=79 / 977,8.1 \%)\end{array}$ & $\begin{array}{l}\text { CURB-65 3-5 } \\
(n=142 / 740,19.2 \%)\end{array}$ & $\begin{array}{l}\text { Total } \\
(n=257 / 3523,7.3 \%)\end{array}$ \\
\hline Streptococcus pneumoniae & $15 / 290(5)$ & $7 / 180(4)$ & 23/142 (16) & $45 / 613(7)$ \\
\hline Haemophilus influenzae & $0 / 32(0)$ & $0 / 23(0)$ & 2/15 (13) & 2/70 (3) \\
\hline Atypical bacteria & $1 / 182(1)$ & $3 / 58(5)$ & $0 / 23(0)$ & $4 / 263(2)$ \\
\hline Legionella pneumophila & $0 / 65(0)$ & $2 / 36(6)$ & $0 / 17(0)$ & $2 / 118(2)$ \\
\hline Mycoplasma pneumoniae & $1 / 55(2)$ & 1/7 (14) & $0 / 3(0)$ & $2 / 65(3)$ \\
\hline Virus & $1 / 81(1)$ & $1 / 43(2)$ & $1 / 24(4)$ & $3 / 148(2)$ \\
\hline Staphylococcus aureus & $1 / 8(13)$ & 2/11 (18) & $3 / 6(50)$ & 6/25 (24) \\
\hline MS & $1 / 2(50)$ & 1/7 (14) & $2 / 5(40)$ & 4/14 (29) \\
\hline MR & $0 / 6(0)$ & $1 / 4(25)$ & $1 / 1(100)$ & 2/11 (18) \\
\hline GNEB & $1 / 10(10)$ & $2 / 4(50)$ & 5/13 (39) & $8 / 27(30)$ \\
\hline
\end{tabular}

Data are expressed as number of deaths/n (\%).

GNEB, Gram-negative enteric bacilli; MR, methicillin-resistant; MS, methicillin-sensitive.

pyogenic bacteria were associated. ${ }^{24}$ In patients with CAP due to mixed infections, the attending clinicians correctly recognised this aetiology as a moderate- to high-risk condition whereas both severity scores were less sensitive in this regard. Only PSI showed some trend in this direction. Since severity scoring was always present in clinical decision making, clinicians possibly overruled the predictions made by the scores. However, it remains unclear on what basis they did so. This issue may require separate analysis.

Only two previous studies have tried to assess the association of severity score and aetiology. In a limited number of patients $(\mathrm{n}=533)$ Roson et al found only $S$ pneumoniae, GNEB, aspiration and unknown aetiologies to be associated with increased severity (PSI class V) and mortality. However, the analysis is limited by small numbers with a definite aetiology $(n=283)$. Dambrava et al assessed the related aetiology according to American Thoracic Society risk stratification and found Legionella spp., viruses and mixed infections to be more frequent in high-risk groups and atypical bacterial pathogens other than Legionella spp. in low-risk groups. Interestingly, with the exception of the very low-risk group (previously healthy, no risk factors) in which pneumococci were somewhat less frequently encountered, $S$ pneumoniae was found to an equal extent in all risk groups. Again, the number of patients studied $(n=829)$ and aetiologies defined (39\%) was limited. Nevertheless, several of these preliminary findings, although not expressively interpreted in the context of our approach, are supported by this analysis.

Our results do not provide further clues into the intriguing observation that both severity scores-albeit of overall equal predictive potential-obviously perform different individual classifications. All that can be said from our analysis is that both scores reflect the aetiology to a very similar extent, and that the aetiology does not seem to be a factor behind the observed divergent individual predictions. Since CURB-65 is far easier to apply, our findings support the use of this score as recommended in the most recent British Thoracic Society (BTS) guideline update. ${ }^{8}$

Our results also complement the CAP aetiological data review recently provided by the BTS guideline update $e^{8}$ in two respects: (1) they support the importance and similar frequency of $L$ pneumophila in any clinical setting (ambulatory, ward and
ICU); and (2) they confirm the relatively high frequency of severe CAP mixed pneumonias, a finding of potential importance for new empirical antibiotic recommendations.

An important limitation of our study is that the microbiological assessment was not homogeneous over time, which limits the validity of the microbial aetiology patterns found. This is particularly true for antigen testing. However, in studies which include long study periods such changes may be inevitable.

In conclusion, CAP due to atypical bacterial pathogens is recognised both clinically and by severity scoring as a low-risk condition and L pneumophila may be correctly identified as less severe than was thought in the past in the era of antigen testing. GNEB (in our study mainly Escherichia coli) and $P$ aeruginosa are correctly identified as moderate- and high-risk pathogens, although prognostic considerations in patients with these aetiologies might lead to different treatment allocation. Mixed aetiologies are conditions that may be underestimated by severity scores.

The criteria behind the clinical recognition of severity in patients with mixed aetiologies remain unclear but, if identified, mixed aetiologies should probably be added as an independent risk factor. The excess mortality of mixed aetiologies supports initial empirical antimicrobial combination treatment, at least in patients with severe CAP.

Funding CibeRes (CB06/06/0028)-ISCiii, 2009 SGR 911 and IDIBAPS.

\section{Competing interests None.}

Ethics approval It was not considered necessary to obtain ethical approval as this is a non-interventional study based on an epidemiological database of prospectively and routinely collected data.

Provenance and peer review Not commissioned; externally peer reviewed.

\section{REFERENCES}

1. Steinhoff D, Lode H, Ruckdeschel G, et al. Chlamydia pneumoniae as a cause of community-acquired pneumonia in hospitalized patients in Berlin. Clin Infect Dis 1996;22:958-64

2. Jokinen C, Heiskanen $\mathrm{L}$, Juvonen $\mathrm{H}$, et al. Microbial etiology of community-acquired pneumonia in the adult population of 4 municipalities in eastern Finland. Clin Infect Dis 2001;32:1141-54.

3. Ruiz M, Ewig S, Marcos MA, et al. Etiology of community-acquired pneumonia: impact of age, comorbidity, and severity. Am J Respir Crit Care Med 1999;160:397-405 
4. Fine MJ, Auble TE, Yealy DM, et al. A prediction rule to identify low-risk patients with community-acquired pneumonia. N Engl J Med 1997;336:243-50.

5. Lim WS, van der Eerden MM, Laing $R$, et al. Defining community acquired pneumonia severity on presentation to hospital: an international derivation and validation study. Thorax 2003;58:377-82.

6. Mandell LA, Wunderink RG, Anzueto A, et al. Infectious Diseases Society of America/American Thoracic Society consensus guidelines on the management of community-acquired pneumonia in adults. Clin Infect Dis 2007:44(Suppl 2):S27-72.

7. Woodhead M, Blasi F, Ewig S, et al. Guidelines for the management of adult lower respiratory tract infections. Eur Respir J 2005;26:1138-80.

8. Lim WS, Baudouin SV, George RC, et al. BTS guidelines for the management of community acquired pneumonia in adults: update 2009. Thorax 2009;64(Suppl 3): iii1-55.

9. Roson B, Carratala J, Dorca J, et al. Etiology, reasons for hospitalization, risk classes, and outcomes of community-acquired pneumonia in patients hospitalized on the basis of conventional admission criteria. Clin Infect Dis 2001;33:158-65.

10. Dambrava PG, Torres A, Valles $\mathrm{X}$, et al. Adherence to guidelines' empirical antibiotic recommendations and community-acquired pneumonia outcome. Eur Respir $J$ 2008;32:892-901.

11. Altman DG. Practical Statistic for Medical Research. 1st edn. London: Chapman and Hall, 1991:403-9.

12. Ewig S, de Roux A, Bauer $T$, et al. Validation of predictive rules and indices of severity for community acquired pneumonia. Thorax 2004:59:421-7.

13. Capelastegui A, Espana PP, Quintana JM, et al. Validation of a predictive rule for the management of community-acquired pneumonia. Eur Respir J 2006:27:151-7.

14. Aujesky D, Auble TE, Yealy DM, et al. Prospective comparison of three validated prediction rules for prognosis in community-acquired pneumonia. Am J Med 2005;118:384-92.
15. Ewig S, Birkner N, Strauss R, et al. New perspectives on community-acquired pneumonia in 388406 patients. Results from a nationwide mandatory performance measurement programme in healthcare quality. Thorax 2009;64:1062-9.

16. Lim WS, Macfarlane JT. A prospective comparison of nursing home acquired pneumonia with community acquired pneumonia. Eur Respir J 2001;18:362-8.

17. von Baum H, Welte T, Marre R, et al. Mycoplasma pneumoniae pneumonia revisited within the German Competence Network for Community-acquired pneumonia (CAPNETZ). BMC Infect Dis 2009;9:62.

18. Vergis EN, Indorf A, File TM Jr, et al. Azithromycin vs cefuroxime plus erythromycin for empirical treatment of community-acquired pneumonia in hospitalized patients: a prospective, randomized, multicenter trial. Arch Intern Med 2000:160:1294-300.

19. Benin AL, Benson RF, Besser RE. Trends in Legionnaires disease, 1980-1998: declining mortality and new patterns of diagnosis. Clin Infect Dis 2002:35:1039-46.

20. von Baum H, Ewig S, Marre R, et al. Community-acquired Legionella pneumonia: new insights from the German competence network for community acquired pneumonia. Clin Infect Dis 2008;46:1356-64.

21. Arancibia $\mathbf{F}$, Bauer TT, Ewig $S$, et al. Community-acquired pneumonia due to gramnegative bacteria and Pseudomonas aeruginosa: incidence, risk, and prognosis. Arch Intern Med 2002;162:1849-58.

22. Jennings LC, Anderson TP, Beynon KA, et al. Incidence and characteristics of vira community-acquired pneumonia in adults. Thorax 2008:63:42-8.

23. von Baum H, Welte T, Marre R, et al. Community-acquired pneumonia through enterobacteriaceae and pseudomonas aeruginosa: diagnosis, incidence and predictors. Eur Respir J 2010;35:598-605.

24. de Roux A, Ewig S, Garcia E, et al. Mixed community-acquired pneumonia in hospitalised patients. Eur Respir J 2006;27:795-800.

\section{Lung alert}

\section{Tobacco-specific carcinogen NNK has an important role in lung cancer}

Lung cancer is the leading cause of mortality in industrialised countries and $85-90 \%$ of lung cancers are caused by cigarette smoking. DNA methyltransferase 1 (DNMT1), an enzyme that catalyses DNA methylation, is reported to be overexpressed in patients with lung and liver cancer who are smokers. The tobacco-specific carcinogen NNK also induces DNA methylation. Unravelling how NNK induces DNMT1-mediated promoter hypermethylation will improve the understanding of tobacco carcinogenesis and may provide an important link between tobacco smoking and lung cancer.

In this study, using lung cancer as a model, animal and clinical studies were undertaken to analyse the molecular mechanisms of DNMT1 overexpression in relation to NNK. DNMT1 overexpression strongly correlated with smoking status and poorer prognosis in patients with lung cancer. NNK was found to increase DNMT1 protein expression and activity by prolonging DNMT1 protein stability through AKT signalling. NNK treatment induced DNMT1 accumulation in the nucleus and in mouse lung adenoma tissues.

These findings provide evidence for the relevance of DNMT1 overexpression in tobacco carcinogenesis and help explain how DNMT1 overexpression may serve as a prognostic factor in smoking-related cancer. Drugs aimed at DNMT1 depletion may prove to be a good therapeutic strategy in the future.

Lin RK, Hsieh YS, LinP, et al. The tobacco-specific carcinogen NNK induces DNA methyltransferase 1 accumulation and tumor suppressor gene hypermethylation in mice and lung cancer patients. J Clin Invest 2010;120:521-32.

\section{Leanne Burke}

Correspondence to Leanne Burke, York Hospitals NHS Foundation Trust, York, UK; leanneburke@doctors.org.uk

Published Online First 13 October 2010

Thorax 2011;66:346. doi:10.1136/thx.2010.145631 